\title{
3 Research Square

\section{Artemisinin attenuated oxidative stress and apoptosis by inhibiting autophagy in MPP+-treated SH-SY5Y cell}

Junqiang Yan ( $\square$ yanjq@haust.edu.cn )

First Affiliated Hospital of Henan University of Science and Technology https://orcid.org/0000-00026489-2824

\section{Hongxia Ma}

Henan Institute of Science and Technology

Xiaoyi Lai

Henan Institute of Science and Technology

Jiannan Wu

Henan Institute of Science and Technology

Anran Liu

Henan Institute of Science and Technology

Jiarui Huang

Henan Institute of Science and Technology

Wenjie Sun

Henan Institute of Science and Technology

Mengmeng Shen

Henan Institute of Science and Technology

Yude Zhang (D835378697@qq.com )

Henan Institute of Science and Technology

Research

Keywords: Artemisinin, Parkinson's disease, Oxidative Stress, Apoptosis, Autophagy

Posted Date: September 9th, 2020

DOI: https://doi.org/10.21203/rs.3.rs-64841/v1

License: (c) (1) This work is licensed under a Creative Commons Attribution 4.0 International License.

Read Full License 
Version of Record: A version of this preprint was published at Journal of Biological ResearchThessaloniki on February 25th, 2021. See the published version at https://doi.org/10.1186/s40709-02100137-6. 


\section{Abstract \\ Background}

Parkinson's disease (PD) is the second most common neurodegenerative disease after Alzheimer's. The drugs currently used to treat PD cannot inhibit the development of PD, and long-term use produces severe drug resistance and adverse reaction. Artemisinin (ART) is an active ingredient of Artemisia annua and has a neuroprotective effect, but the mechanism is still unclear. This study was designed to investigate the neuroprotective effect of ART in MPP'-treated SH-SY5Y cells.

\section{Results}

There was no significant cytotoxicity when the ART concentration was under. $40 \mu \mathrm{M}$. The $20 \mu \mathrm{M}$ ART for $24 \mathrm{~h}$ could increase the cell viability by reducing oxidative stress and cell apoptosis in MPP${ }^{+}$-treated $\mathrm{SH}^{-}$ SY5Y cell. In addition, immunoblot and immunofluorescence results showed that MPP ${ }^{+}$treatment increased the expression of Beclin1, LC3II/LC3I and decreased the expression of P62, while ART can reverse the changes caused by $\mathrm{MPP}^{+}$.

\section{Discussion}

More and more researches reported that ART and its derivates have neuroprotective effects through antioxidant and anti-apoptosis. we found that pre-treated cells with $20 \mu \mathrm{M}$ ART for $4 \mathrm{~h}$ could significantly increase the viability in Parkinson's disease cell model. The oxidative stress and apoptosis were the main reason for the degeneration of dopaminergic neurons, while artemisinin can attenuate oxidative stress and apoptosis in MPP $\mathrm{MP}^{+}$-lesioned dopaminergic neurons. The levels of autophagy proteins LC3II/I, Beclin1 and P62 also showed that MPP + increased the autophagy level, and pre-treatment with ART decreased the autophagy level, which may be the pathological mechanism for artemisinin to reduce oxidative stress damage and apoptosis.

\section{Conclusions}

These results indicate that ART exerts a positive effect on MPP+-treated SH-SY5Y cells in terms of antioxidative stress and anti-apoptosis. These effects may be related to autophagy. These findings contribute to a better understanding of the critical role of ART in PD treatment.

\section{Introduction}

Parkinson's disease (PD) is a neurodegenerative disease characterized by degeneration and death of dopamine (DA) neurons in the substantia nigra of the midbrain ${ }^{1}$, with a prevalence more than $1 \%{ }^{2}$. The 
main manifestations of PD include resting tremor, bradykinesia, muscle rigidity and gait disturbance ${ }^{3}$. At present, drugs used to treat PD, such as dopamine agonists and L-DOPA, can alleviate the symptoms of $\mathrm{PD}$, but they cannot inhibit the development of PD, and long-term use produces severe drug resistance and adverse reactions ${ }^{4}$. Therefore, studying the pathogenesis of Parkinson's disease and finding new drugs that can inhibit DA neuron apoptosis and inflammation have great significance for the treatment of PD.

It has been reported that oxidative stress, apoptosis and autophagy play an important role in the pathogenesis of $\mathrm{PD}^{5-7}$. Therefore, drugs acting on these mechanisms may become promising approaches for the treatment of PD. Oxidative stress is a negative effect produced by free radicals in the body and is considered to be an important factor leading to disease and aging. Studies suggested that oxidative stress might be one of the core aspects of PD pathogenesis ${ }^{8,9}$, under various harmful factors, oxidative stress damage to the DA neurons in the midbrain substantia nigra, followed by mitochondrial dysfunction, finally activating the apoptotic signal pathway, starting the programmed cell death process, and eventually causing nervous system dysfunction. Autophagy is the process of engulfing self-damaged proteins or organelles and sending them into lysosomes for degradation and reuse ${ }^{10}$. The function of autophagy is reflected in that it is important for cells to maintain their own homeostasis and metabolic balance. In adverse environments such as oxidative stress, the body initiates various of defense mechanisms, such as increasing the levels of antioxidants, degrading specific misfolded proteins through the ubiquitin-proteasome system, and degrading damaged proteins and organelles through autophagy. Studies have shown that autophagy plays an important role in protecting body from cancer, infection, neurodegeneration, type 2 diabetes, aging and so on ${ }^{11-13}$.

Artemisinin(ART), extracted from the stems and leaves of Artemisia annua, has been used as an antimalarial drug for a long time and have saved millions of lives ${ }^{14,15}$. However, with the deepening of research, other effects of ART have been discovered and applied, such as anti-tumor, anti-diabetes, antioxidant, anti-apoptotic, immunoregulation and so on ${ }^{16-18}$. Recent years, studies have reported that ART has neuroprotective effect through anti-oxidative stress and anti-apoptosis ${ }^{19-21}$. Furthermore, study of ART in another neurodegenerative disease, Alzheimer's disease, have been reported. Sarina et $\mathrm{al}^{22}$ reported that ART protects PC12 cells from $\beta$-amyloid-induced apoptosis by activating the ERK $1 / 2$ signaling pathway. However, the protective effect of ART on the mode of PD has not been studied so far. So, in this study, we try to investigate the neuroprotective effects of ART in an in vitro model of PD and attempt to reveal its potential molecular mechanisms.

\section{Materials And Methods}

\section{Cell culture and drug treatment}

Human neuroblastoma cell line SH-SY5Y was obtained from Sun Ye San University (Guangzhou, China), and cultured in DMEM/H medium (Hyclone, Logan, UT, USA) with $10 \%$ fetal bovine serum(Gibco, Grand 
Island, NY, USA ) and $1 \%$ glutamine at $37^{\circ} \mathrm{C}$ in a humidified incubator with $5 \% \mathrm{CO} 2$. We changed the culture medium every other two days, and subcultured the cells when the density reached $80 \%$. (1) To study the effect of $\mathrm{MPP}^{+}$or ART on cell viability, we treated the cells with different concentrations of $\mathrm{MPP}^{+}$or ART for 24h and performed CCK-8 assay; (2) To study the protective effect of ART on MPP+induced cytotoxicity, we treated the cells with PBS, ART, MPP ${ }^{+}, \mathrm{ART}^{+} \mathrm{MPP}^{+}$as indicated concentrations for $24 \mathrm{~h}$. ART $(20 \mu \mathrm{M})$ pre-treated the cells for $4 \mathrm{~h}$, and then added $\mathrm{MPP}^{+}$for $24 \mathrm{~h}$. (3) To study the changes of

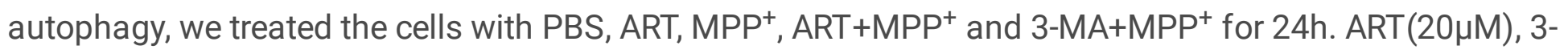
MA ( $5 \mathrm{mM})$ pre-treated the cells for $4 \mathrm{~h}$, and then added $\mathrm{MPP}^{+}$for $24 \mathrm{~h}$. MPP ${ }^{+}$was purchased from Sigma (CAS No. 36913-39-0, USA). ART was purchased from DASF (CAS No. 71963-77-4, Nanjing, China). Autophagy inhibitor 3-methyladenine (3-MA) was purchased from sigma (M9281, USA).

\section{Cell viability}

Cell viability was measured by CCK-8 assay (Solarbio, CA1210, China) according to the manufacturer's instructions. Briefly, SH-SY5Y cells were seeded in 96-wells culture plates for $24 \mathrm{~h}$, and treated with MPP or ART for another $24 \mathrm{~h}$, then $100 \mu \mathrm{l}$ culture medium containing $10 \mathrm{mM}$ CCK-8 was added to each well and incubated at $37^{\circ} \mathrm{C}$ for $2 \mathrm{~h}$. The absorbance at $450 \mathrm{~nm}$ was measured by a multi-mode microplate reader (EnSpire, PerkinElmer, Singapore). The cell viability of the control group was set to $100 \%$, and the cell viability of the other groups were compared with that of the control group.

\section{Intracellular Reactive Oxygen Species (ROS) assay}

Levels of intracellular ROS were measured by ROS assay kit (Beyotime Biotechnology, S0033, China) according to the manufacturer's instructions. After drug treatment, $\mathrm{SH}-\mathrm{SY} 5 \mathrm{Y}$ cells were incubated with serum-free fresh medium which was contained $10 \mu \mathrm{M}$ DCFH-DA at $37^{\circ} \mathrm{C}$ for $20 \mathrm{~min}$, then washed the cells with serum-free culture medium for three times. Add $1 \mathrm{ml}$ fresh medium into each well, the fluorescence was observed using the GFP channel by an inverted fluorescence microscopy (Nikon, Japan) and the fluorescence intensity was analyzed by ImageJ.

\section{Superoxide dismutase (SOD) activity assay}

Intracellular SOD activity was measured by total SOD activity detection kit (Beyotime Biotechnology, S0101, China) according to the manufacturer's instructions. After drug treatment, washed the cells once with PBS, and centrifuged to collect the cells. Lysed the cells fully with SOD sample preparation solution and collected the supernatants at $12,000 \times \mathrm{g}$ for $5 \mathrm{~min}$ at $4^{\circ} \mathrm{C}$. Detected their absorbance at $450 \mathrm{~nm}$ and $600 \mathrm{~nm}$ (reference wavelength) by a multi-mode microplate reader and calculated the SOD activity.

\section{Glutathione (GSH) assay}

The content of GSH was measured by the glutathione assay kit (Beyotime Biotechnology, S0052, China) according to the manufacturer's instructions. After drug treatment, washed the cells once with PBS, and centrifuged to collect the cells. Prepared the samples and standards and detected their absorbance at 
$412 \mathrm{~nm}$. Made the standard curve and calculated the contents of GSH in the samples according to the standard curve.

\section{Malondialdehyde (MDA) assay}

Intracellular MDA levels were measured by MDA detection kit (Beyotime Biotechnology, S0131, China) according to the manufacturer's instructions. After drug treatment, collected the cells. Lysed the cells and collected the supernatants at $10,000 \times \mathrm{g}$ for $10 \mathrm{~min}$. Prepared the standards, then detected the absorbance of samples and standards at $532 \mathrm{~nm}$ and $450 \mathrm{~nm}$ (reference wavelength). The contents of MDA in the samples were quantified based on the standard curve.

\section{Mitochondrial membrane potential (MMP) assay}

To monitor mitochondrial integrity, mitochondrial membrane potential assay kit with JC-1 (Beyotime Biotechnology, C2006, China) was used according to manufacturer's instructions. Briefly, after drug treatment, SH-SY5Y cells were incubated with $\mathrm{JC}-1$ working solution for at $37^{\circ} \mathrm{C} 20 \mathrm{~min}$ and washed twice with JC-1 buffer. Then observed the red fluorescence and green fluorescence through the GFP channel and TRITC channel by an inverted fluorescence microscopy (Nikon, Japan), and the fluorescence intensity was analyzed by ImageJ.

\section{Western blot assay}

Cell lysates were prepared and the protein concentrations were quantified with BCA protein assay kit (CWBIO, CW2011S, China). Equal protein samples were separated wite 12\% SDS-PAGE and transferred to PVDF membranes. After blocked with $5 \%$ skim milk at room temperature for $1 \mathrm{~h}$, the membranes were incubated at $4^{\circ} \mathrm{Covernight}$ with primary antibody (1:1000) against cleaved caspase-3 (Cell Signaling Technology, Aps175, USA), caspase-3 (Abcam, ab90437, UK), LC3 (Abcam, ab128025, UK), Beclin1(BD, 612113, USA), P62(Abcam, ab56416, UK) or $\beta$-actin 1:5000 (CWBIO, CW0096M, China). The next day, membranes were washed with TBST for three times, and incubated with HRP-conjugated anti-rabbit or anti-mouse secondary antibody (CWBI0,1:5000) at room temperature for $1 \mathrm{~h}$. The blots were detected by cECL western blot kit (CWBIO, CW0049M, China) and images were analyzed by ImageJ.

\section{Immunofluorescence Staining}

SH-SY5Y cells were seeded on slides in 12-well culture plates. After drug treatment, cells were fixed with ice methanol for $5 \mathrm{~min}$, and blocked with $1 \%$ bovine serum albumin (BSA) for $30 \mathrm{~min}$, then incubated with primary antibody against LC3(1:200), P62(1:100) respectively at $4^{\circ} \mathrm{C}$ for overnight. The next day, slides were incubated with Alexa Fluor 488 (Bioss Antibodies, bs-0295G-AF488, China) or Alexa Fluor 555 (Bioss Antibodies, bs-0296G-AF555, China) secondary antibody $(1: 500)$ at $37^{\circ} \mathrm{C}$ for $1 \mathrm{~h}$ and incubated with DAPI (BOSTER, AR1177) for $5 \mathrm{~min}$. The fluorescence was observed by a fluorescence microscope (OLYMPUS, $\mathrm{BX53).}$

\section{Statistical Analysis}


Results were expressed as the mean \pm standard error of mean (mean \pm SEM). Comparison among multiple groups were performed with One-way ANOVA followed by Tukey post- hoc test and $\mathrm{P}<0.05$ was considered statistically significant.

\section{Results}

\section{ART attenuated MPP+-induced cytotoxicity in SH-SY5Y cells}

We treated the cells with different concentrations of $\mathrm{MPP}^{+}(0.2,0.4,0.8,1.0 \mathrm{mM})$ for $24 \mathrm{~h}$ and found that $\mathrm{MPP}^{+}$could significantly reduce the cell viability and had dose-dependent (Fig.1a). Next, we treated the cells with different concentrations of ART $(2.5,5,10,20,40 \mu \mathrm{M})$ for $24 \mathrm{~h}$, the results showed that there was no obvious cytotoxicity when the concentrations reached $40 \mu \mathrm{M}(\mathrm{Fig} .1 \mathrm{~b})$. Then, we treated the cells with various concentrations of $\operatorname{ART}(5,10,20,40 \mu \mathrm{M})$ for $4 \mathrm{~h}$ before treated with $1 \mathrm{mM} \mathrm{MPP}^{+}$, the results showed that cell viability increased first and then decreased compared with the $\mathrm{MPP}^{+}$group, cell viability reached maximum when the ART concentration reached $20 \mu \mathrm{M}$ (Fig.1c).

\section{ART reduced MPP+-induced oxidative stress in SH-SY5Y cells}

We investigated the effect of ART on MPP+-induced oxidative stress injury in SH-SY5Y cells by detecting the ROS production, SOD activity, GSH levels and MDA levels. As shown in Fig.2a and 2b, the ROS production was increased to $2.48 \pm 0.20$ after treated with MPP ${ }^{+}$which had an obviously difference with the control $1.00 \pm 0.13$. However, after pre-treatment with ART, the ROS production was markedly reduced to $1.67 \pm 0.16$ compared with $\mathrm{MPP}^{+}$.

As shown in Fig. $2 \mathrm{c}$ and $2 \mathrm{~d}$, after exposed to MPP ${ }^{+}$, the levels of SOD and GSH were obviously reduced from $29.9 \pm 2.15 \mathrm{U} / \mathrm{mg}$ to $17.7 \pm 1.53 \mathrm{U} / \mathrm{mg}$ and from $6.5 \pm 0.5 \mu \mathrm{mol} / \mathrm{mg}$ to $3.23 \pm 0.31 \mu \mathrm{mol} / \mathrm{mg}$ compared with their corresponding control group. However, after pre-treatment with ART, the levels of SOD and GSH markedly increased from $17.7 \pm 1.53 \mathrm{U} / \mathrm{mg}$ to $23.3 \pm 2.08 \mathrm{U} / \mathrm{mg}$ and from $3.23 \pm 0.31 \mu \mathrm{mol} / \mathrm{mg}$ to $4.9 \pm 0.36 \mathrm{U} / \mathrm{mg}$ compared with their corresponding $\mathrm{MPP}^{+}$group.

As shown in Fig.2e, the content of MDA was obviously increased to $1.24 \pm 0.13 \mathrm{nmol} / \mathrm{mg}$ in $\mathrm{MPP}^{+}$group, which was only $0.61 \pm 0.13 \mathrm{nmol} / \mathrm{mg}$ in the control group. After pre-treatment with ART, the content of MDA significantly reduced to $0.91 \pm 0.09 \mathrm{nmol} / \mathrm{mg}$ compared with $\mathrm{MPP}^{+}$group.

\section{ART alleviated MPP+-induced Mitochondrial membrane potential (MMP) damage in SH-SY5Y cells}

$\mathrm{JC}-1$ is an ideal fluorescent probe and is widely used to detect MMP, and the transition of JC-1 from red fluorescence to green fluorescence can be used as an indicator of early apoptosis. As shown in Fig. 3, In normal cells and ART-treated cells, JC-1 staining showed bright red fluorescence, and weak green fluorescence. After SH-SY5Y cells were treated with $\mathrm{MPP}^{+}$for $24 \mathrm{~h}, \mathrm{MMP}$ decreased significantly, showing a decrease of red fluorescence and an increase of green fluorescence. However, pre-treatment with ART 
could reduce the dissipation of MMP compared with $\mathrm{MPP}^{+}$, showing an increase of red fluorescence and a decrease of green fluorescence.

\section{ART reduced MPP+ ${ }^{+}$-induced apoptosis in SH-SY5Y cells}

To investigate whether ART has an anti-apoptotic effect on MPP ${ }^{+}$-induced SH-SY5Y cells, we detected the expression of caspase-3, cleaved caspase-3 and performed hoechst33258 staining and flow cytometry analysis. As the results showed $\mathrm{MPP}^{+}$significantly increased the relative expression of cleaved caspase-3 compared with control group, however, pre-treatment with ART, the expression of cleaved caspase-3 significantly reduced compared with $\mathrm{MPP}^{+}$group (Fig.4a).

\section{ART inhibited the autophagy induced by MPP ${ }^{+}$}

In order to study the effect of ART on autophagy in MPP+-induced SH-SY5Y cells, we detected the expression of Beclin-1, P62 and LC3 which were the maker proteins of autophagy. As the western blot showed (Fig. 5a), compared with the control group, MPP ${ }^{+}$significantly increased the beclin- 1 expression and the conversion of LC3I to LC3II, but decreased the expression of P62, these indicated that MPP ${ }^{+}$could induced autophagy. However, pre-treatment with ART, the expression of Beclin-1 and the conversion of LC3I to LC3II were decreased, and the expression of P62 was increased significantly. Interestingly, the regulation of ART on autophagy maker proteins had the same trend as that of 3-MA in MPP ${ }^{+}$-induced SHSY5Y cells. These results suggested that ART could inhibite autophagy induced by MPP ${ }^{+}$.

To verify above results, we performed immunofluorescence staining. As the resultes showed (Fig. 5b), $\mathrm{MPP}^{+}$significantly increased the LC3 expression and decreased the P62 expression compared with control group, however, pre-treatment with ART, the expression of LC3 was decreased and the expression of P62 was increased compared with $\mathrm{MPP}^{+}$group. These results were consistent with the results of western blot.

\section{Discussion}

ART, a sesquiterpene lactone, is the best drug for the treatment of malaria, and the combination therapy based on it is also the most effective and important method for treating malaria ${ }^{23}$. Recently, more and more researches reported that ART and its derivates have neuroprotective effects through anti-oxidant and anti-apoptosis. Therefore, we study the neuroprotective effect of ART in MPP ${ }^{+}$-induced SH-SY5Y cell model. Firstly, we studied the toxicity of ART and found that there was no significant cytotoxicity when the ART concentration reached $40 \mu \mathrm{M}$, and this dose was also within the range of clinically safe doses approved by the FDA ${ }^{24}$. Next, we found that pre-treated cells with $20 \mu \mathrm{M}$ ART for $24 \mathrm{~h}$ could significantly increase the viability of MPP+treated SH-SY5Y cells. Therefore, we chose $20 \mu \mathrm{M}$ ART in this study.

A large number of studies have confirmed that oxidative stress damage is the main reason for the degeneration of dopaminergic neurons and the relationship between oxidative stress and PD has been 
the focus of many scholars. Futhermore, studies have found that there is obvious oxidative stress phenomenon in the substantia nigra of PD patients ${ }^{25,26}$. $\mathrm{MPP}^{+}$, as a neurotoxin closely related to the pathogenesis of $\mathrm{PD}$, can lead to mitochondrial dysfunction and oxidative stress, and ultimately lead to neuronal death. Our results also proved that: $\mathrm{MPP}^{+}$reduced the mitochondrial membrane potential, inducesd oxidative stress damage, and leaded to apoptosis in SH-SY5Y cells, which were consistent with previous studies. More notably, we found that ART could protect SH-SY5Y cells from oxidative stress injury and apoptosis induced by MPP ${ }^{+}$. To our best knowledge, our study revealed for the first time that ART exerted neuroprotective effects in the cell model of PD. Therefore, ART might be a potential neuronprotective drug in PD, and may have important reference value for clinical treatment of PD.

Recent research believes that autophagy is a double-edged sword with dual functions. On the one hand, it can protect cells by degrading abnormal proteins and organelles. On the other hand, if autophagy is overactive or low function, it will form a autophagic stress to damages organelles, such as causing mitochondrial dysfunction, etc., causing cell damage $\mathrm{e}^{10,27}$. Similarly, autophagy also plays a dual role in responding to oxidative stress. When the body responds to oxidative stress damage, the activation of autophagy can facilitate the survival of cells by removing oxidized proteins and damaged organelles ${ }^{28}$. However, on the other hand, with the continuous enhancement of autophagy, it can directly induce the death of oxidized cells and further damage to mitochondria, thereby reversely aggravating the oxidative stress damage of cells ${ }^{29}$. By monitoring the levels of autophagy proteins LC3II/I, Beclin1 and P62, we found that MPP ${ }^{+}$increased the autophagy level, and pre-treatment with ART decreased the autophagy level compared with $\mathrm{MPP}^{+}$. However, increased levels of autophagy have been reported in neurodegenerative diseases including $\mathrm{PD}^{30,31}$. Our results supported the previous researches that autophagy involved in the occurrence and development of $\mathrm{PD}^{32,33}$.

The following are some limitations of our study: (1) All studies were conducted under in vitro conditions. Although $\mathrm{MPP}^{+}$is commonly used to prepare the in vitro model of $\mathrm{PD}, \mathrm{MPP}^{+}$-induced in vitro model of PD was not exactly the same as the microenvironment of dopaminergic neurons in vivo. (2) Our experiments confirm that ART could reduce oxidative stress damage and excessive autophagy caused by MPP ${ }^{+}$, but the relationship between ART in reducing oxidative stress damage and its regulation of autophagy was worthy of our further research.

\section{Conclusion}

In conclusion, our results indicated that ART reduces $\mathrm{MPP}^{+}$-induced neurotoxicity in cell model of PD by reducing oxidative stress, apoptosis and inhibiting autophagy. At the same time, we revealed a new therapeutic effect of ART and this might provide new hope for preventing and delaying PD in the future.

\section{Declarations}

Ethics approval and consent to participate 
Not applicable

\section{Consent for publication}

Not applicable

\section{Availability of data and materials}

All data generated or analysed during this study are included in this published article

\section{Competing interests}

The authors declare that they have no competing interests

\section{Funding}

Medical science and technology research in Henan Province(LHGJ20190560)

\section{Authors' contributions}

Hongxia Ma and Xiaoyi Lai were involved in the manuscript preparation. Jiarui Huang, Mengmeng Shen and Anran Liu were involved in accessing datas. Jiarui Huang and Wenjie Sun involved in checking proofreading. Junqiang Yan and Yude Zhang was involved in design, writing and organization).

\section{Acknowledgements}

Not applicable

\section{References}

1. Liu W, et al. Long non-coding RNA MALAT1 contributes to cell apoptosis by sponging miR-124 in Parkinson disease. Cell Biosci. 2017;7:19. doi:10.1186/s13578-017-0147-5.

2. Dexter DT, Jenner P. Parkinson disease: from pathology to molecular disease mechanisms. Free Radic Biol Med. 2013;62:132-44. doi:10.1016/j.freeradbiomed.2013.01.018.

3. Scheperjans F, et al. Gut microbiota are related to Parkinson's disease and clinical phenotype. Mov Disord. 2015;30:350-8. doi:10.1002/mds.26069.

4. Jang W, et al. The Neuroprotective Effect of Erythropoietin on Rotenone-Induced Neurotoxicity in SHSY5Y Cells Through the Induction of Autophagy. Mol Neurobiol. 2016;53:3812-21. doi:10.1007/s12035-015-9316-x.

5. Zhu J, et al. Apelin-36 mediates neuroprotective effects by regulating oxidative stress, autophagy and apoptosis in MPTP-induced Parkinson's disease model mice. Brain Res. 2020;1726:146493. doi:10.1016/j.brainres.2019.146493. 
6. Baluchnejadmojarad T, Mansouri M, Ghalami J, Mokhtari Z, Roghani M. Sesamin imparts neuroprotection against intrastriatal 6-hydroxydopamine toxicity by inhibition of astroglial activation, apoptosis, and oxidative stress. Biomed Pharmacother. 2017;88:754-61. doi:10.1016/j.biopha.2017.01.123.

7. Cherra SJ 3rd \& Chu CT. Autophagy in neuroprotection and neurodegeneration: A question of balance. Future Neurol. 2008;3:309-23. doi:10.2217/14796708.3.3.309.

8. Gorman AM, McGowan A, O'Neill C, Cotter T. Oxidative stress and apoptosis in neurodegeneration. Journal of the neurological sciences. 1996;139 Suppl:45-52. doi:10.1016/0022-510x(96)00097-4.

9. Chi H, Guan Y, Li F, Chen Z. The Effect of Human Umbilical Cord Mesenchymal Stromal Cells in Protection of Dopaminergic Neurons from Apoptosis by Reducing Oxidative Stress in the Early Stage of a 6-OHDA-Induced Parkinson's Disease Model. Cell Transplant. 2019;28:87S-99S. doi:10.1177/0963689719891134.

10. Klionsky DJ, Emr SD. Autophagy as a regulated pathway of cellular degradation. Science. 2000;290:1717-21. doi:10.1126/science.290.5497.1717.

11. Xie Q, Liu M, Yan YF, Shen X, Wang ES. Exogenous Tetranectin Protects Against 1-Methyl-4Phenylpyridine-Induced Neurotoxicity by Inhibiting Apoptosis and Autophagy Through Ribosomal Protein S6 Kinase Beta-1. World Neurosurg. 2019;122:e375-82. doi:10.1016/j.wneu.2018.10.058.

12. Tuohetaerbaike $B$, et al. Pancreas protective effects of Urolithin A on type 2 diabetic mice induced by high fat and streptozotocin via regulating autophagy and AKT/mTOR signaling pathway. J Ethnopharmacol. 2020;250:112479. doi:10.1016/j.jep.2019.112479.

13. Onorati AV, Dyczynski M, Ojha R, Amaravadi RK. Targeting autophagy in cancer. Cancer. 2018;124:3307-18. doi:10.1002/cncr.31335.

14. Cui L, Su XZ. Discovery, mechanisms of action and combination therapy of artemisinin. Expert Rev Anti Infect Ther. 2009;7:999-1013. doi:10.1586/eri.09.68.

15. Meshnick SR. Artemisinin: mechanisms of action, resistance and toxicity. Int J Parasitol. 2002;32:1655-60. doi:https://doi.org/10.1016/S0020-7519(02)00194-7.

16. Steely AM, Willoughby JA, Sr., Sundar SN, Aivaliotis VI, Firestone GL. Artemisinin disrupts androgen responsiveness of human prostate cancer cells by stimulating the $26 \mathrm{~S}$ proteasome-mediated degradation of the androgen receptor protein. Anticancer Drugs. 2017;28:1018-31. doi:10.1097/CAD.0000000000000547.

17. Chong $\mathrm{CM}$, Zheng W. Artemisinin protects human retinal pigment epithelial cells from hydrogen peroxide-induced oxidative damage through activation of ERK/CREB signaling. Redox Biol. 2016;9:50-6. doi:10.1016/j.redox.2016.06.002.

18. Lin SP, Li W, Winters A, Liu R, Yang SH. Artemisinin Prevents Glutamate-Induced Neuronal Cell Death Via Akt Pathway Activation. Front Cell Neurosci. 2018;12:108. doi:10.3389/fncel.2018.00108.

19. Zheng W, et al. Artemisinin conferred ERK mediated neuroprotection to PC12 cells and cortical neurons exposed to sodium nitroprusside-induced oxidative insult. Free Radic Biol Med. 2016;97:158-67. doi:10.1016/j.freeradbiomed.2016.05.023. 
20. Zhao X, et al. Artemisinin Attenuated Hydrogen Peroxide (H2O2)-Induced Oxidative Injury in SH-SY5Y and Hippocampal Neurons via the Activation of AMPK Pathway. Int J Mol Sci 20, doi:10.3390/ijms20112680 (2019).

21. Sarina, et al. Induction of neurite outgrowth in PC12 cells by artemisinin through activation of ERK and p38 MAPK signaling pathways. Brain Res. 2013;1490:61-71. doi:10.1016/j.brainres.2012.10.059.

22. Zeng Z, Xu J, Zheng W. Artemisinin protects PC12 cells against beta-amyloid-induced apoptosis through activation of the ERK1/2 signaling pathway. Redox Biol. 2017;12:625-33. doi:10.1016/j.redox.2017.04.003.

23. Visser BJ, van Vugt M, Grobusch MP. Malaria: an update on current chemotherapy. Expert Opin Pharmacother. 2014;15:2219-54. doi:10.1517/14656566.2014.944499.

24. Yan F, Wang H, Gao Y, Xu J, Zheng W. Artemisinin Protects Retinal Neuronal Cells against Oxidative Stress and Restores Rat Retinal Physiological Function from Light Exposed Damage. ACS Chemical Neuroscience. 2017;8:1713-23. doi:10.1021/acschemneuro.7b00021.

25. Reichmann H, et al. Unaltered Respiratory Chain Enzyme Activity and Mitochondrial DNA in Skeletal Muscle from Patients with Idiopathic Parkinson's Syndrome. Eur Neurol. 1994;34:263-7. doi:10.1159/000117053.

26. Gatt AP, et al. Dementia in Parkinson's disease is associated with enhanced mitochondrial complex I deficiency. Mov Disord. 2016;31:352-9. doi:10.1002/mds.26513.

27. Martinet W, Agostinis P, Vanhoecke B, Dewaele M, de Meyer GR. Y. Autophagy in disease: a doubleedged sword with therapeutic potential. Clin Sci. 2009;116:697-712. doi:10.1042/CS20080508.

28. Filomeni G, De Zio D, Cecconi F. Oxidative stress and autophagy: the clash between damage and metabolic needs. Cell Death Differ. 2015;22:377-88. doi:10.1038/cdd.2014.150.

29. Sun J, Yue F. Suppression of REDD1 attenuates oxygen glucose deprivation/reoxygenation-evoked ischemic injury in neuron by suppressing mTOR-mediated excessive autophagy. J Cell Biochem. 2019;120:14771-9. doi:10.1002/jcb.28737.

30. Nixon RA. Autophagy, amyloidogenesis and Alzheimer disease. J Cell Sci. 2007;120:4081. doi:10.1242/jcs.019265.

31. Isidoro C, et al. The Role of Autophagy on the Survival of Dopamine Neurons. Curr Top Med Chem. 2009;9:869-79.

32. Pan T, Kondo S, Le W, Jankovic J. The role of autophagy-lysosome pathway in neurodegeneration associated with Parkinson's disease. Brain. 2008;131:1969-78. doi:10.1093/brain/awm318.

33. Wang $X$, et al. alpha-synuclein promotes progression of Parkinson's disease by upregulating autophagy signaling pathway to activate NLRP3 inflammasome. Exp Ther Med. 2020;19:931-8. doi:10.3892/etm.2019.8297.

\section{Figures}



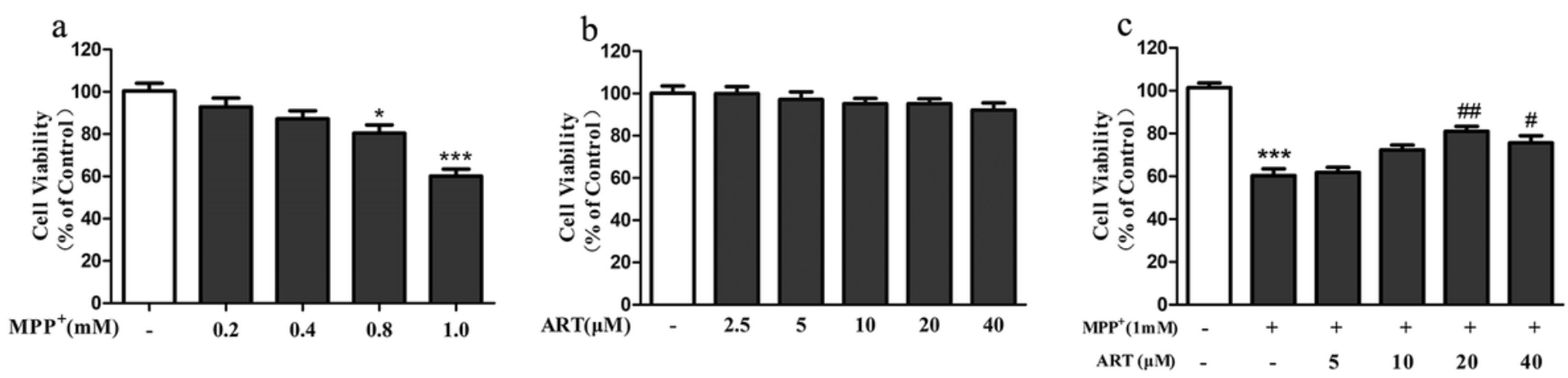

Figure 1

ART reduced MPP+-induced cytotoxicity in SH-SY5Y cells. (a) Effects of different concentrations of MPP+ on the viability of SH-SY5Y cells. (b) Effects of different concentrations of ART on the viability of SHSY5Y cells. (c) Effects of different concentrations of ART on the viability of MPP+-induced SH-SY5Y cells. Results were represented as mean $\pm S D(n=6),{ }^{*} p<0.05,{ }^{* \star} p<0.01$ versus control group; $\# p<0.05 \# \# p<0.01$ versus MPP+ group.

a
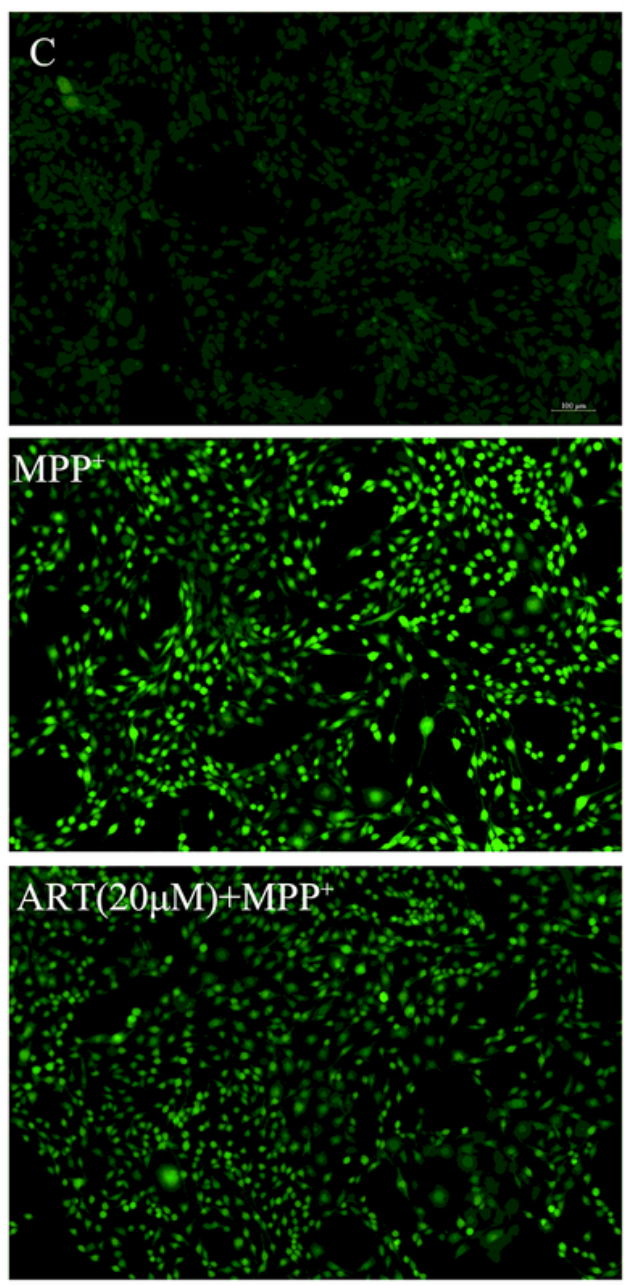
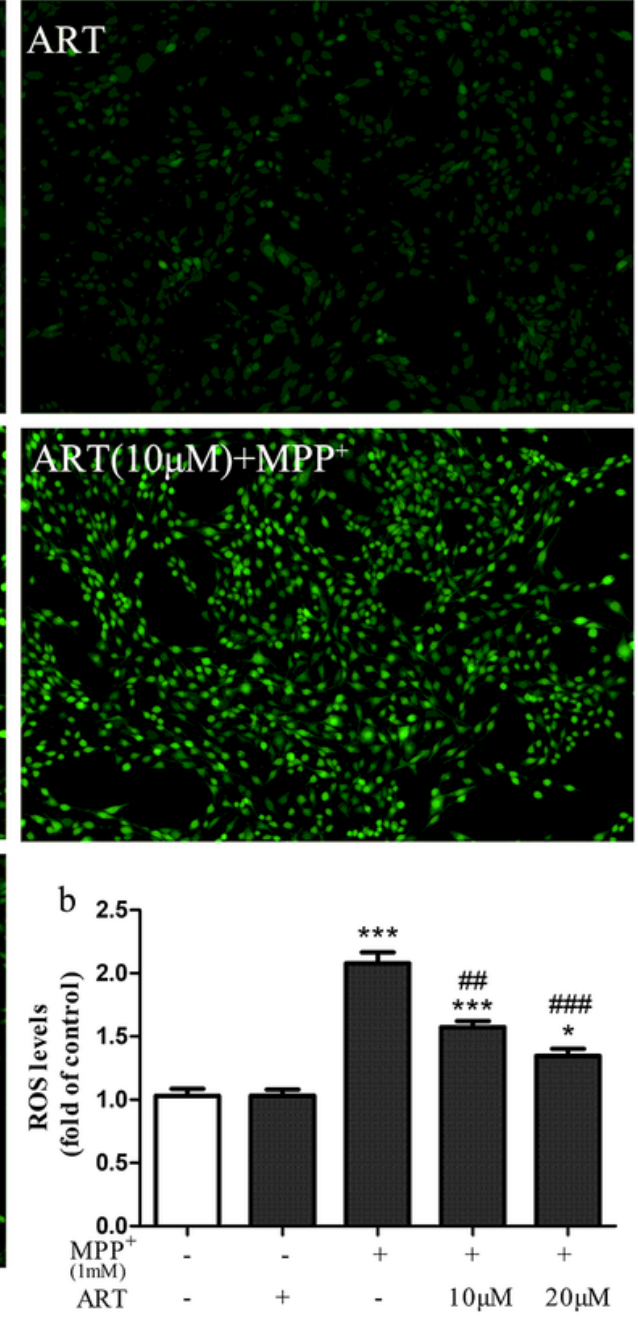
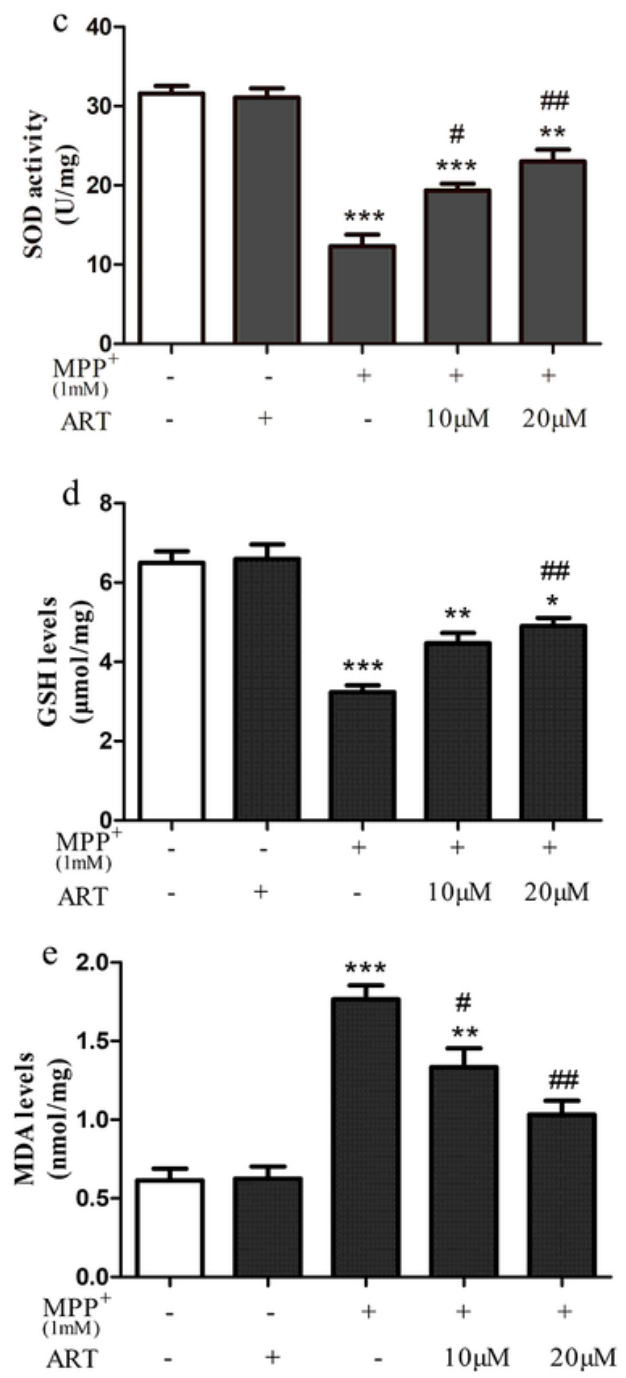


\section{Figure 2}

ART reduced oxidative stress injury induced by MPP+. (a) ART decreased the ROS generation compared with MPP+, (b) represented the fluorescence intensity of (a). ART increased the SOD activities (c) and the GSH production(d), decreased the MDA production (e) compared with MPP+. bar=100 $\mu \mathrm{m}$. Results were represented as means $\pm S D(n=3),{ }^{\star} p<0.05,{ }^{*} p<0.01,{ }^{* \star} \mathrm{p}<0.001$ versus control group; $\# p<0.05 \# \# p<0.01$ versus MPP+ group.

a

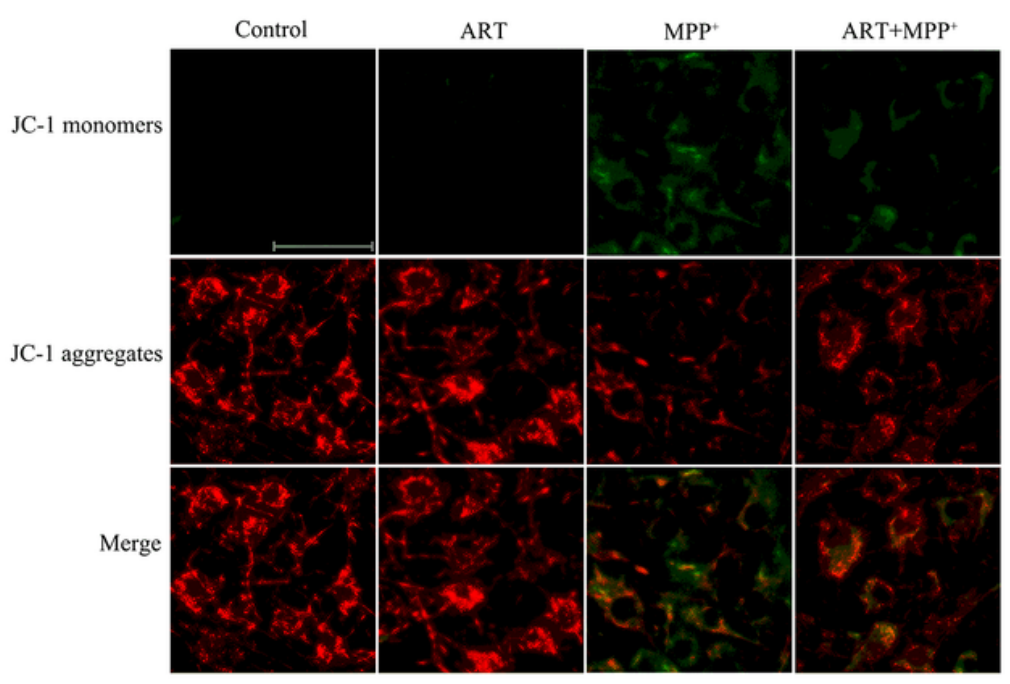

b

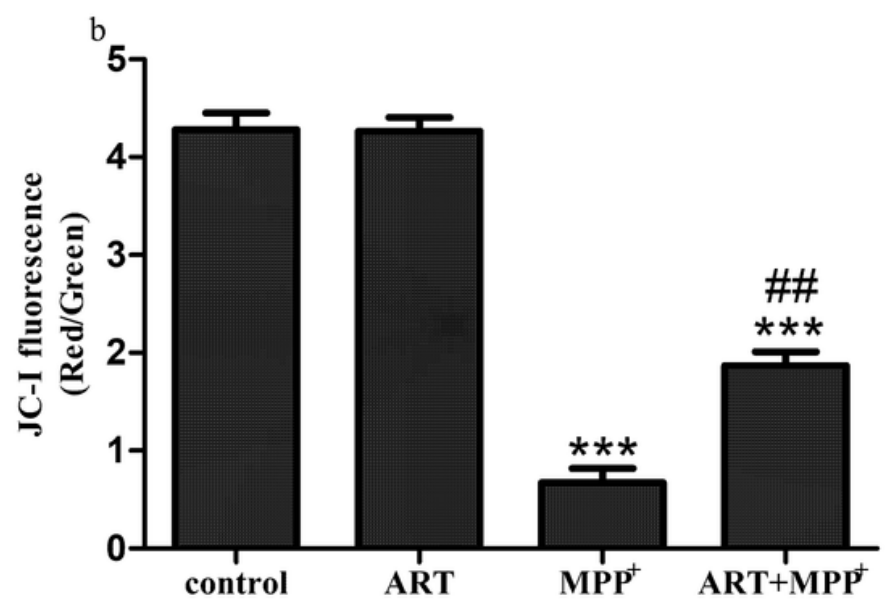

Figure 3

ART alleviates MPP+-induced mitochondrial damage. (a)The red fluorescence represented JC-1 aggregates and indicated high MMP. The green fluorescence represented the JC-1 monomers and indicated low MMP. (b)The ratio of red fluorescence intensity to green fluorescence intensity was counted and the results were represented as means $\pm S D(n=3)$. bar $=50 \mu m .{ }^{\star \star \star} p<0.001$ versus control group; $\# \# p<0.01$ versus $M P P+$ group.
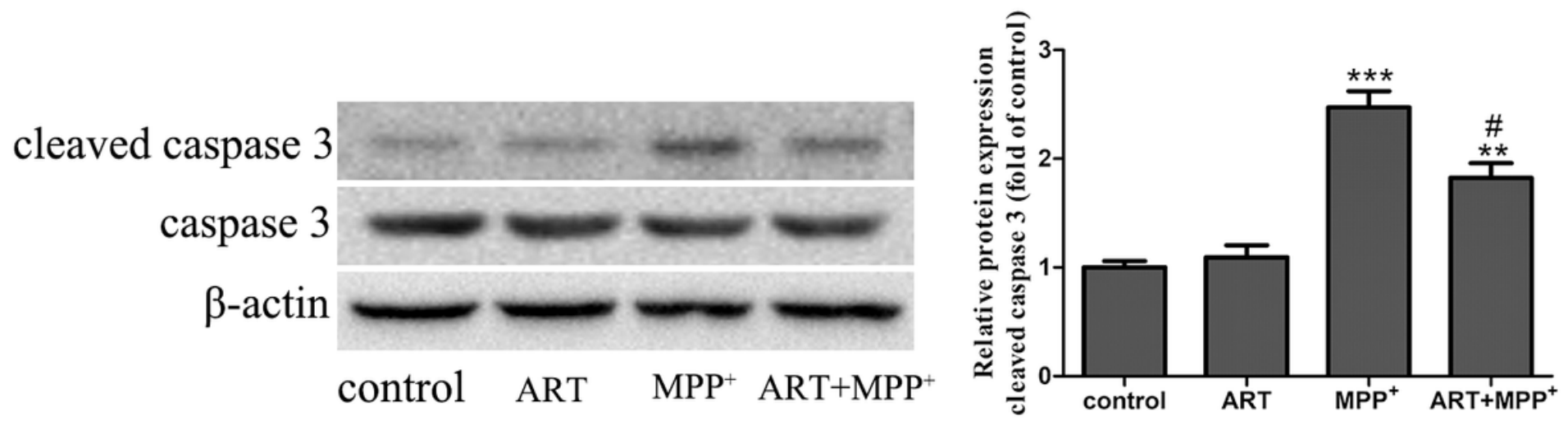

Figure 4

ART reduced MPP+-induced apoptosis in SH-SY5Y cells. (a)The expression of caspase-3 and cleaved caspase-3 were measured using western blot. (b-c) ${ }^{\star *} p<0.01$, $* \star * p<0.001$ versus control group; $\# p<0.05$ 
$\# \# p<0.01$ versus $M P P+$ group.

a

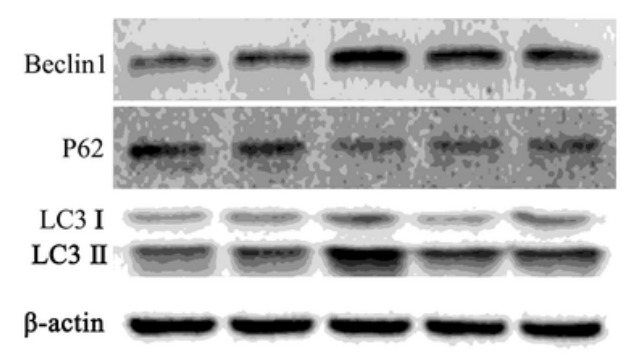

$\begin{array}{llllll}\mathrm{ART} & - & + & - & + & - \\ \mathrm{MPP}^{+} & - & - & + & + & + \\ 3-\mathrm{MA} & - & - & - & - & +\end{array}$

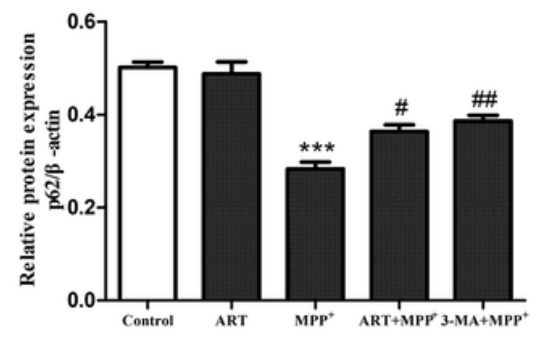

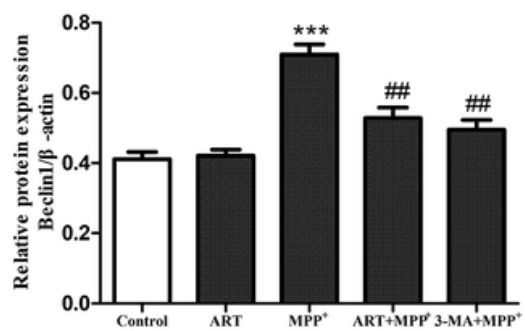

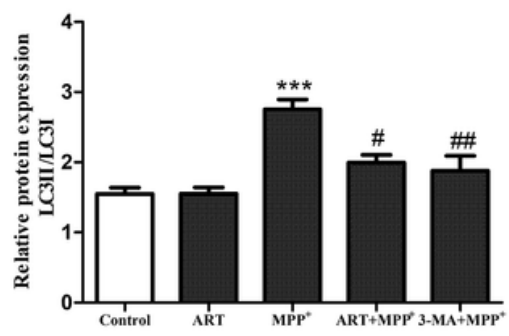

b
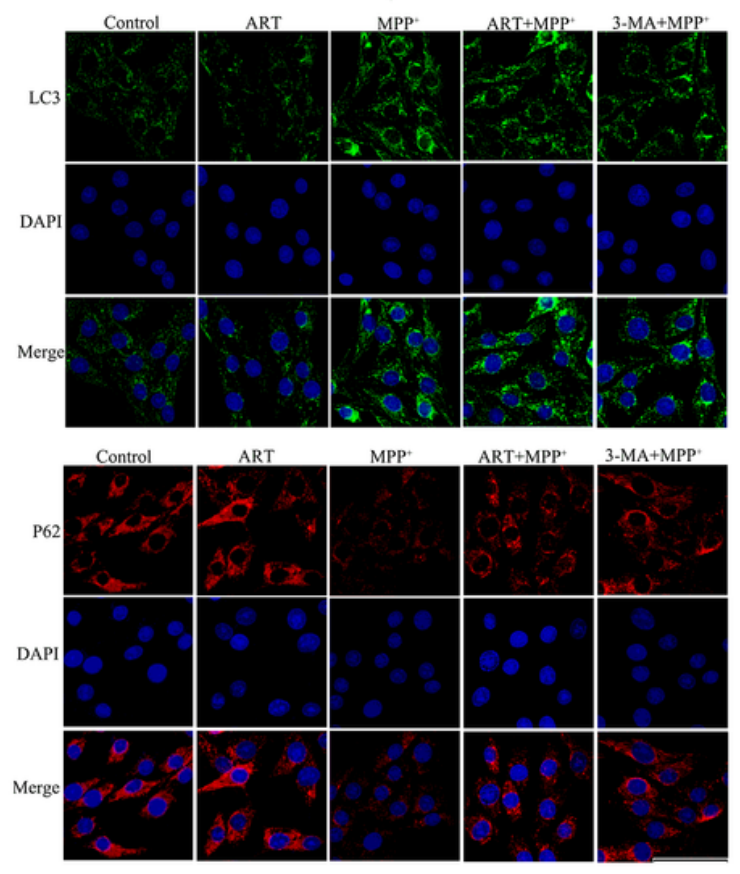

Figure 5

ART inhibited the autophagy induced by MPP+ in SH-SY5Y cells. (a)The protein levels of Beclin-1, P62 and LC3 were determined by Western blot. $\beta$-actin was used as an equal loading control. Data were presented as the mean $\pm S D(n=3)$. bar $=50 \mu m$. ${ }^{* \star *} p<0.001$ versus control group; $\# p<0.05 \# \# p<0.01$ versus MPP+ group. (b)The expression of P62 and LC3 basing on immunofluorescence staining. 